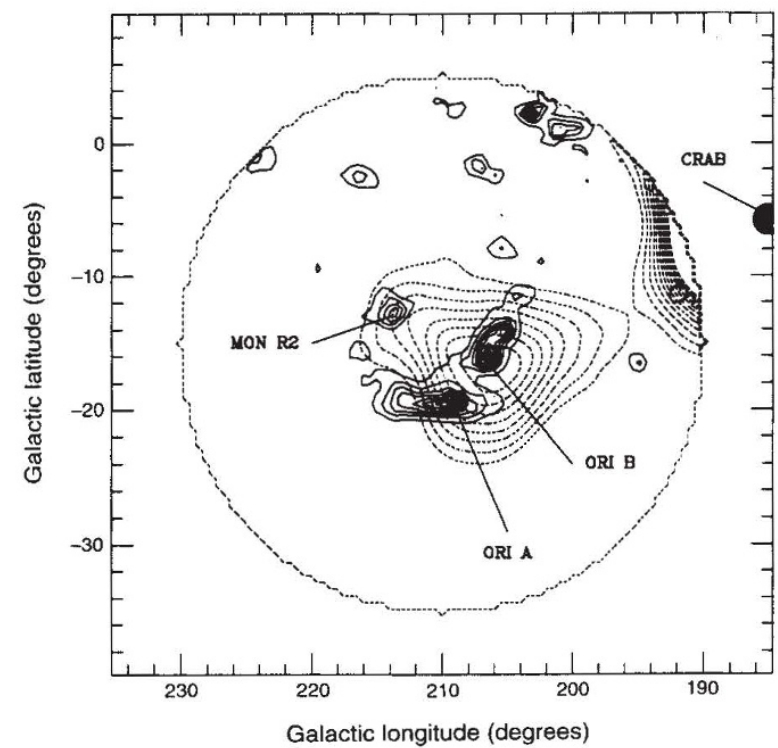

FIG. 2 COMPTEL map of the Orion region in the 3-7-MeV range (dotted contours) identified with the 4.44- and 6.13-MeV de-excitation lines of carbon and oxygen nuclei. The Columbia University carbon monoxide observations are superimposed for comparison (from ref. 7).

each with its own star formation history ${ }^{3}$. Good candidates for these parental fragments are super-massive self-gravitating clouds whose physical properties resemble those of current molecular clouds such as Orion ${ }^{4}$.

Most encouragingly, the processes of enrichment and mixing can now be observed through the direct imaging of nuclear de-excitation $\gamma$-ray lines by instruments such as the COMPTEL telescope on board the Compton Gamma Ray Observatory (H. Bloemen, SRONUtrecht). One example is the radioactive decay of ${ }^{26} \mathrm{Al}$ by positron emission into an excited state of ${ }^{26} \mathrm{Mg}$. This process has a half-life of about a million years and the resultant $1.809-\mathrm{MeV}$ line has now been mapped along the Milky Way (Fig. 1). Although the precise origin of the ${ }^{26} \mathrm{Al}$ is not established yet, the extended and structured appearance of the Milky Way observed at $1.809 \mathrm{MeV}$ suggests that regions where massive stars form play a dominant role through their production of supernova type II ejecta and stellar (Wolf-Rayet) winds. Another important result is the possible COMPTEL detection of nuclear interaction lines of carbon and oxygen nuclei from the Orion complex at 4.44 and $6.13 \mathrm{MeV}$ (Fig. 2).

The patchy nature of the COMPTEL ${ }^{26} \mathrm{Al}$ map suggests that the amount of that isotope $\left(1.5 M_{\odot}\right)$ spread throughout the interstellar medium is a product of nucleosynthesis in massive stars. But the inferred average ratio of ${ }^{26} \mathrm{Al} /{ }^{27} \mathrm{Al}$ in the interstellar medium is still an order of magnitude smaller than the large ratio, 5 $\times 10^{-5}$, found in meteorites that formed in the early solar disk. Clayton ${ }^{5}$ argued that the low-energy cosmic rays responsi- ble for the 4.43-MeV $\gamma$-rays detected by COMPTEL from the Orion clouds are also responsible for the ${ }^{26} \mathrm{Al}$ in molecular cloud cores, as supernova models have difficulty in accounting for this high ratio through selfenrichment. Another possibility is that particles from the enriched hot medium are bombarding the ambient dense gas, after having been accelerated by colliding stellar winds and multiple supernova explosions (Bloemen).

The process of star formation can be studied through the isotopic abundances within meteorites that locked in information about conditions prevalent at the birth of our Solar System. The inferred initial abundances of most of the shorterlived radionuclides such as ${ }^{60} \mathrm{Fe},{ }^{53} \mathrm{Mn}$ and ${ }^{107} \mathrm{Pd}$ (halflife of 6.5 million years) is consistent with a burst of self-enrichment by type II supernovae. The Solar System may therefore have formed in the compressed wall of metal-enriched gas that abuts a region of vigorous $\mathrm{O}$ and $\mathrm{B}$ star formation as is occurring in the Orion molecular cloud.

The presence of long-lived isotopes such as ${ }^{129} \mathrm{I}$ (half-life of 16 million years) is also very important (C. L. Harper Jr, Harvard Univ.). Thus, whereas the shorttimescale data indicate an episode of self-enrichment of the parental cloud, the inference from the data on iodine with its longer decay time is that the parental cloud underwent a long period of isolation from type II bursts. This could occur naturally for parental gas that was overtaken by a spiral arm of the Galaxy. The denser regions of high-mass star formation and type II supernovae in the spiral arms would produce spikes in the ${ }^{129} \mathrm{I}$ record followed by long quiet spells while the cloud was between arms (C. L. Harper, manuscript submitted).

The Orion region may not only be presenting us with a picture of our own violent origins. It may also be a laboratory for star-formation processes that occurred when our Galaxy was assembling.

Ralph E. Pudritz is in the Department of Physics and Astronomy, McMaster University, Hamilton, Ontario L8S 4M1. Canada.

1. Bolte, M. ASP Conf. Proc. 48,60 (1993).

2. Wheeler, J. C., Sneden, C. \& Truran, J. W. Jr. A. Rev. Astr. Astrophys. 27, 279(1989)

3. Searie, L. \& Zinn, R. Astrophys. J. 225, 357 (1978)

4. Harris, W. E. \& Pudritz, R. E. Astrophys. J. 429, 177 (1994)

5. Clayton, D. D. Nature 368, 222 (1994)

6. Dieht, R. et al. Astr. Astrophys. (in the press)

7. Bloemen, H. et al. Astr. Astrophys. 281, L5 (1994)

\section{Navigation grid}

LIKE most developed regions, Europe is covered with a dense power grid. It carries gigawatts of $50 \cdot \mathrm{Hz}$ alternating current through lines often hundreds of kilometres long. The resulting a.c. magnetic field must extend around the lines for some function of this characteristic distance. It must blanket the whole continent, and even reach up into space. Daedalus now has a use for it. He sees it as a huge distributed European radar aerial.

Unlike conventional radar, it would work not by radiation but by induction. The grid acts as the primary winding of a vast transformer. Any metal object within its field - such as an aircraft at a height of a few kilometres - acts as a secondary winding: a current is induced in it. This in turn perturbs the field. By measuring the field at many points, it should be possible to detect this perturbation and deduce its source. To complete the radar, therefore, a set of sensing wires is needed. The grid pylons themselves could easily carry an extra wire or so, and many other existing conductors could be pressed into service. Telephone lines, data links, wire fences, railway signal cables, motorway dividing strips and so on, all could pick up their local $50 \cdot \mathrm{Hz}$ field and monitor its direction, amplitude and phase. These measurements could be relayed back to a central computer, and compared with the currents flowing at that moment within the grid itself. In the absence of air traffic, they would define the background field of the continent. If aircraft now flew over the system, they would perturb the field. Subtracting the known unperturbed field would reveal these perturbations, and locate the singularities from which they emanated: the aircraft.

For air-traffic control, this system has many advantages. Europe could replace its many control centres with one big network. It could be extended into any other region which had a 50-Hz grid kept in phase with the European one. Aircraft using the system could enhance their visibility by carrying tuned coil-andcapacitor $50 . \mathrm{Hz}$ resonators, but even Stealth bombers should show up clearly enough. Indeed, for air defence the continental hum field would be ideal. It is everywhere all the time, it is too big and widely distributed to be knocked out, it gives away no information of its own, and nothing can fly under or over it. The only snag is that it would detect surface traffic as well. This could overload the computation. But, says Daedalus, the low speed and defined tracks of surface traffic make it easy to recognize and subtract from the perturbation analysis at an early stage. David Jones 